This item was submitted to Loughborough's Research Repository by the author.

Items in Figshare are protected by copyright, with all rights reserved, unless otherwise indicated.

\title{
Regulation of magnetically actuated satellites using model predictive control with disturbance modelling
}

PLEASE CITE THE PUBLISHED VERSION

PUBLISHER

(C) IEEE

LICENCE

CC BY-NC-ND 4.0

REPOSITORY RECORD

Wood, Mark, and Wen-Hua Chen. 2008. "Regulation of Magnetically Actuated Satellites Using Model Predictive Control with Disturbance Modelling”. figshare. https://hdl.handle.net/2134/3967. 
This item was submitted to Loughborough's Institutional Repository (https://dspace.lboro.ac.uk/) by the author and is made available under the following Creative Commons Licence conditions.

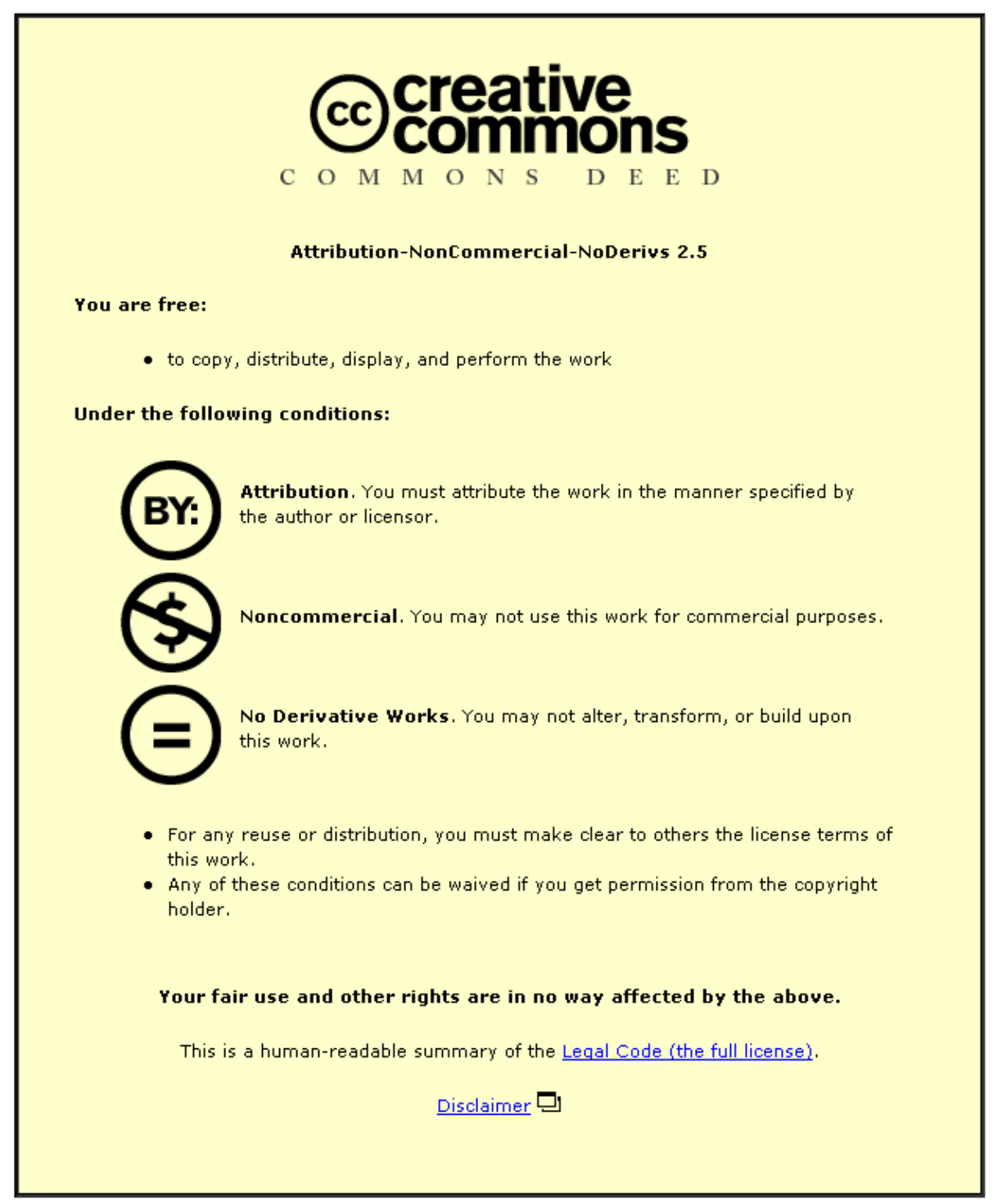

For the full text of this licence, please go to: http://creativecommons.org/licenses/by-nc-nd/2.5/ 


\title{
Regulation of Magnetically Actuated Satellites using Model Predictive Control with Disturbance Modelling
}

\author{
Mark Wood and Wen-Hua Chen
}

\begin{abstract}
The problem of magnetic attitude regulation of low Earth orbiting satellites is addressed using model predictive control. This paper considers extending the current literature within this area by combining a standard predictive controller with a suitable disturbance model. For low Earth orbiting satellites the disturbances due to the external environment are very significant and consideration of these torques within the control law is imperative to obtain the best possible controller performance. In order to obtain an estimate of the external disturbance, a state estimator of varying complexity is used. This estimator initially assumes that the disturbances acting on the satellite remain constant with time, but this assumption is then improved to assume harmonic variation of the disturbance. Simulations show that inclusion of a feed-forward element to the control offers notable performance benefits. Interestingly, increasing the complexity of the disturbance model seems to offer little improvement in performance and a simple constant disturbance model provides a suitable solution to the problem while maintaining simplicity of the state estimator.
\end{abstract}

\section{INTRODUCTION}

$\mathrm{T}$ HE area of spacecraft magnetic attitude control is one that has attracted much recent attention in the research literature. Use of magnetic dipoles to control the attitude of a spacecraft offers a lightweight, smooth and cost-effective method of control. Although this is the case, the torque generated through use of magnetic dipoles is constrained to lie in the plane orthogonal to the local magnetic field vector, with one axis being instantaneously under-actuated. If the satellite is on an inclined orbit, suitable variation of the magnetic field allows controllability in the long term, but presents a significant challenge from a control perspective.

Several different approaches to the magnetic control problem have been proposed by different authors, varying in type and complexity. Perhaps the most basic approach suggested is use of a simple PD controller. The control signal is calculated as if full controllability is available and this 'ideal' control signal is then projected onto the plane orthogonal to the magnetic field vector for implementation. This type of approach is suggested by several authors, with main contributions in [1]-[3]. Although PD control is a simple

This work was supported in part by the European Space Agency.

Mark Wood is a Research Student in the Department of Aeronautical and Automotive Engineering at Loughborough University, UK. (E-mail: M.Wood@lboro.ac.uk)

Wen-Hua Chen is a Senior Lecturer in flight control systems in the Department of Aeronautical and Automotive Engineering at Loughborough University, UK. (E-mail: W.Chen@lboro.ac.uk) approach to the problem, it cannot always give the desired performance. As a result several authors try to exploit the pseudo-periodic nature of the Earth's magnetic field to use periodic linear quadratic control theory. Reference [4] presents such an approach, with the resulting control law depending on the solution of the time-varying Ricatti equation. Under certain assumptions, the solution to the Ricatti equation tends to a constant value and hence the final controller simplifies to a full state feedback controller, with the only time variance of the problem appearing from the magnetic field vector. Similar infinite horizon LQR approaches are also considered in [5] and [6].

Model predictive control (MPC) has recently been demonstrated as a suitable candidate for magnetic attitude regulation. Reference [7] considers a novel predictive approach, where the problem is formed in a time-invariant manner, with the time-variance reintroduced through an appropriate set of constraints. This work is extended in [8] to consider practical issues such as the effect of magnetic field modelling. Reference [9] considers a finite horizon controller for removal of large initial Euler angles and demonstrates significant energy savings over other non-linear controllers.

Low Earth orbiting satellites are subject to significant disturbances from the external environment. These disturbances often dominate the response of the attitude dynamics and consideration of these torques and their effects is very important when designing an attitude regulation controller. Attempts to account for these disturbances within an infinite horizon LQR formulation are proposed in [5] and [6], however the use of disturbance modelling within a finite horizon controller has yet to be investigated. With the performance achievable using model predictive control shown to be impressive (see [8]), inclusion of a disturbance model within the predictive control scheme has the potential to further improve performance.

This paper considers the benefits of including a disturbance model within a predictive control scheme. This seeks to quantify the benefits achievable through such an approach, while also investigating the effects of varying the model complexity. This paper is structured as follows. Section 2 introduces the spacecraft attitude dynamics, with a linear model being sufficient to accurately describe the attitude dynamics. Section 3 introduces the model predictive 
approach, with Section 4 introducing the proposed disturbance models and the Kalman filter design. Section 5 considers performance under the two disturbance models and compares this to the case when no disturbance compensation is used.

\section{SPACECRAFT ATTITUDE DYNAMICS}

\section{A. Linearized Attitude Dynamics}

The attitude dynamics of an Earth orbiting satellite are fully described by a series of non-linear differential equations. Under certain assumptions these equations can be linearized with minimal loss of accuracy. If linearization is carried out about the Earth pointing attitude, assuming a circular orbit, small Euler angles and deviation of body rates from nominal values, the following model can be produced (see [4]).

$$
\dot{x}(t)=A_{c} x(t)+B_{c} T(t)
$$

$$
\begin{aligned}
A_{c} & =\left[\begin{array}{cccccc}
0 & 0 & 0 & 1 & 0 & 0 \\
0 & 0 & 0 & 0 & 1 & 0 \\
0 & 0 & 0 & 0 & 0 & 1 \\
-4 \omega_{0}^{2} \sigma_{1} & 0 & 0 & 0 & 0 & \omega_{0}\left(1-\sigma_{1}\right) \\
0 & 3 \omega_{0}^{2} \sigma_{2} & 0 & 0 & 0 & 0 \\
0 & 0 & \omega_{0}^{2} \sigma_{3} & -\omega_{0}\left(1+\sigma_{3}\right) & 0 & 0
\end{array}\right] \\
B_{c} & =\left[\begin{array}{c}
0_{3,3} \\
J^{-1}
\end{array}\right] x=\left[\begin{array}{llllll}
\phi & \theta & \psi & \omega_{x} & \omega_{y} & \omega_{z}
\end{array}\right]^{T}
\end{aligned}
$$

where,

$\phi, \theta, \psi$ are the spacecraft pointing angles about roll, pitch and yaw axes respectively,

$\omega_{x}, \omega_{y}, \omega_{z}$ are the spacecraft angular rates about roll, pitch and yaw axes respectively,

$\omega_{0}$ is the orbital frequency,

$J$ is the satellite inertia matrix of the form $J=\operatorname{diag}\left(\left[\begin{array}{lll}J_{x} & J_{y} & J_{z}\end{array}\right]\right)$,

$J_{x}, J_{y}, J_{z}$ are the principal inertia values about roll, pitch and yaw axes respectively,

$T$ is the control torque applied to the satellite,

$$
\sigma_{1}=\frac{J_{y}-J_{z}}{J_{x}} \quad \sigma_{2}=\frac{J_{z}-J_{x}}{J_{y}} \quad \sigma_{3}=\frac{J_{x}-J_{y}}{J_{z}} .
$$

Note that the co-ordinate system used throughout this paper defines the spacecraft orientation relative to a local-level coordinate system. The local-level system has the $+z$ axis pointing towards the nadir, the $y$ axis perpendicular to the orbital plane, and the $\mathrm{x}$ axis defined by the right hand rule.
The expression $0_{m, n}$ is used frequently throughout this paper to represent a matrix of zeros with $m$ rows and $n$ columns.

\section{B. Actuator Dynamics}

Although the dynamics are accurately described in the previous section, the control torque that can be applied using magnetic control is constrained to lie in the plane perpendicular to the Earth's magnetic field. The magnetic dipole strength and the corresponding control torque are linked through (2).

$$
T=M \times B_{m a g}
$$

Where $B_{\text {mag }}$ is the Earth's magnetic field vector and $M$ is the vector of magnetic dipole moments.

Note that this restriction can also be enforced by selecting the control torque such that the following expression holds

$$
B_{m a g} \cdot T=0
$$

\section{External Disturbance Torques}

There are several environmental torques that affect the dynamics of an Earth orbiting satellite. For satellites operating on a low Earth orbit the upper atmosphere induces aerodynamic forces that in turn lead to disturbance torques within the attitude dynamics. The aerodynamic forces also cause a drag component against the direction of the orbital motion that, if unaccounted for, would cause decay of the satellite orbit. Onboard thrusters are required to counteract this torque and, if offset from the drag line, further disturb the satellite dynamics. Gravity gradient torques unaccounted for in the linearization process can be treated as external torques, while residual magnetic dipoles also further disturb the spacecraft attitude. Such a wide range of disturbances illustrates the need to take account of these within the controller design.

\section{MODEL PREDICTIVE CONTROL}

\section{A. The MPC Approach}

Model predictive control is an advanced control strategy based around an internal model of the plant under control. This model is used to predict the future expected behavior of the plant, with the resulting information being used to determine the 'optimal' control input to be applied. A typical MPC strategy adopts the following basic procedure.

- A mathematical model is used to predict the future satellite behavior over a finite future time period known as the prediction horizon. 
- This predicted behavior can be compared to a desired reference trajectory, with an error vector being generated.

- The optimum control sequence is defined by minimizing the predicted error over the prediction horizon.

- The first input in the 'optimal' control sequence is applied to the plant, with the remaining sequence being discarded and the whole process then repeated at the next sampling interval.

\section{B. Control Problem Formulation}

The linear system in (1) can be described by the discretetime formulation in (4). Note that the control variable $T$ in (1) is substituted for $u$ for more conventional MPC notation, but both represent the control torque.

$x(k+1)=A x(k)+B(u(k)+d(k))$

where $A=\exp \left(A_{c} T_{s}\right), B=\left[\int_{0}^{T_{s}} \exp \left(A_{c} \eta\right) d \eta\right] B_{c}$,

$T_{s}$ is the controller sampling time and $d(k)$ represents the disturbance due to the external environment.

Equation (4) can be iterated over a finite future period to provide predictions of the state vector in terms of the future control sequence.

$X=\left[\begin{array}{c}A \\ A^{2} \\ A^{3} \\ \vdots \\ A^{N}\end{array}\right] x(k)+\left[\begin{array}{ccccc}B & 0 & 0 & 0 & 0 \\ A B & B & 0 & 0 & 0 \\ A^{2} B & A B & B & 0 & 0 \\ \vdots & \vdots & \ddots & \ddots & \vdots \\ A^{N-1} B & A^{N-2} B & A^{N-3} B & \cdots & B\end{array}\right](U+D)^{(5)}$ where $X=\left[\begin{array}{llll}\hat{x}(k+1) & \hat{x}(k+2) & \cdots & \hat{x}(k+N)\end{array}\right]^{T}$, $U=\left[\begin{array}{llll}u(k) & u(k+1) & \cdots & u(k+N-1)\end{array}\right]^{T}$, $D=\left[\begin{array}{llll}\hat{d}(k) & \hat{d}(k+1) & \cdots & \hat{d}(k+N-1)\end{array}\right]^{T}$,

$X$ is the predicted future state vector, $U$ is the future control sequence, $D$ is the vector of predicted disturbances and $N$ is the prediction horizon.

Once the predictions have been carried out the optimal control sequence can be defined by minimizing a suitable performance index. This is typically in a quadratic form as shown below

$$
V=\sum_{i=1}^{N} \hat{x}(k+i)^{T} Q \hat{x}(k+i)+u(k+i-1)^{T} R u(k+i-1)
$$

where $Q$ and $R$ are the state and control weighting matrices respectively.

This optimization can be carried out efficiently using quadratic programming, for which efficient algorithms exist. Note that the minimization must be carried out subject to the constraints imposed by the magnetic actuation, hence

$$
B_{\text {mag }}(k+i) \cdot u(k+i)=0
$$

\section{CONTROLLER DESIGN}

\section{A. Kalman Filter}

An important part of the controller design is providing estimates of the disturbances due to the external environment. The benchmark satellite is equipped with star sensors and accelerometers but the external disturbances and spacecraft angular rates must be estimated using a Kalman filter. Two models are provided to model the external disturbances. Equation (8) assumes the disturbances acting on the satellite are constant with time, while (9) assumes the disturbances are harmonic at the orbital frequency.

$$
\begin{aligned}
& \dot{d}(t)=\left[\begin{array}{lll}
0 & 0 & 0
\end{array}\right]^{T}+v_{c_{1}}(t) \\
& {\left[\begin{array}{c}
\dot{d}(t) \\
\dot{s}(t)
\end{array}\right]=W_{c}\left[\begin{array}{c}
d(t) \\
s(t)
\end{array}\right]+v_{c_{2}}(t)}
\end{aligned}
$$

where $W_{c}=\left[\begin{array}{cc}0 & I_{3} \\ -\omega_{0}^{2} I_{3} & 0\end{array}\right], I_{3}=\left[\begin{array}{lll}1 & 0 & 0 \\ 0 & 1 & 0 \\ 0 & 0 & 1\end{array}\right]$,

$d(t)$ is the disturbance, $s(t)$ is the disturbance derivative and $v_{c_{1}}(t)$ and $v_{c_{2}}(t)$ represent system noise.

As the Kalman filter is to be implemented in discrete-time the equivalent formulations are given as follows.

$$
\begin{aligned}
& d(k+1)=d(k)+v_{1}(k) \\
& {\left[\begin{array}{c}
d(k+1) \\
s(k+1)
\end{array}\right]=W\left[\begin{array}{c}
d(k) \\
s(k)
\end{array}\right]+v_{2}(k)}
\end{aligned}
$$

where $W=\exp \left(W_{c} T_{s}\right)$ and $v_{1}(k)$ and $v_{2}(k)$ are the discrete-time equivalence of $v_{c_{1}}(t)$ and $v_{c_{2}}(t)$.

The state vector can now be augmented with the disturbance models to provide two Kalman filter designs. Firstly consider augmenting the constant disturbance model with the satellite dynamics. 


$$
\begin{aligned}
& x_{e_{1}}(k+1)=A_{e_{1}} x_{e_{1}}(k)+B_{e_{1}} u(k)+\left[\begin{array}{c}
0_{6,3} \\
I_{3}
\end{array}\right] v_{1}(k) \\
& y_{e_{1}}(k)=C_{e_{1}} x_{e_{1}}(k)+D_{e_{1}} u(k)+w_{1}(k)
\end{aligned}
$$

where,

$$
\begin{aligned}
& A_{e_{1}}=\left[\begin{array}{cc}
A & B \\
0_{3,6} & I_{3}
\end{array}\right], B_{e_{1}}=\left[\begin{array}{c}
B \\
0
\end{array}\right], x_{e_{1}}(k)=\left[\begin{array}{c}
x(k) \\
d(k)
\end{array}\right]
\end{aligned}
$$

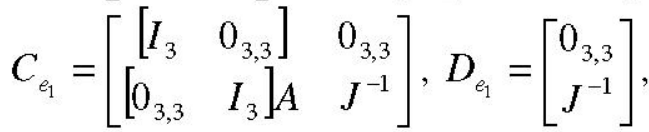

$w_{1}(k)$ is the measurement noise and $y_{e_{1}}(k)$ defines the model outputs of pointing angle and angular acceleration.

An estimate of the augmented state $\hat{x}_{e_{1}}$ can then be provided through the following relationship

$$
\begin{aligned}
\hat{x}_{e_{1}}(k+1) & =A_{e_{1}} \hat{x}_{e_{1}}(k)+B_{e_{1}} u(k) \\
& +L_{e_{1}}\left[y(k)-\left(C_{e_{1}} \hat{x}_{e_{1}}(k)+D_{e_{1}} u(k)\right)\right]
\end{aligned}
$$

$L_{e_{1}}$ is the Kalman filter gain matrix and is chosen to minimize the covariance of the estimation error in the usual way. $y(k)$ is the vector of measured pointing angle and acceleration and is taken directly from star sensor and accelerometer data.

An identical procedure can be followed to develop the Kalman filter with harmonic disturbance model.

$$
\begin{aligned}
& x_{e_{2}}(k+1)=A_{e_{2}} x_{e_{2}}(k)+B_{e_{2}} u(k)+\left[\begin{array}{c}
0_{6,6} \\
I_{6}
\end{array}\right] v_{2}(k) \\
& y_{e_{2}}(k)=C_{e_{2}} x_{e_{2}}(k)+D_{e_{2}} u(k)+w_{2}(k)
\end{aligned}
$$

where,

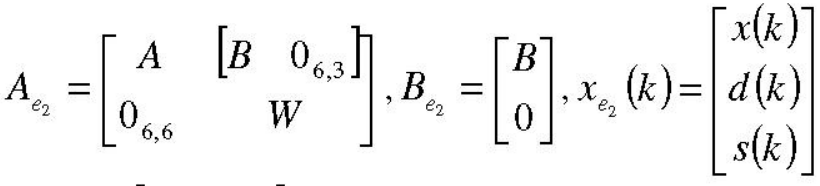

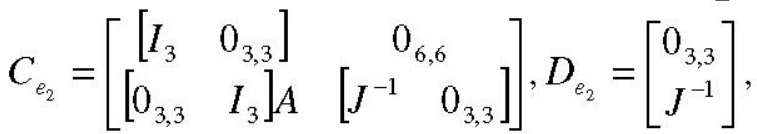

$w_{2}(k)$ is the measurement noise and $y_{e_{2}}(k)$ is the estimated model output.
Once again the augmented state $\hat{x}_{e_{2}}$ can be estimated through the following relationship

$$
\begin{aligned}
\hat{x}_{e_{2}}(k+1) & =A_{e_{2}} \hat{x}_{e_{2}}(k)+B_{e_{2}} u(k) \\
& +L_{e_{2}}\left[y(k)-\left(C_{e_{2}} \hat{x}_{e_{2}}(k)+D_{e_{2}} u(k)\right)\right]
\end{aligned}
$$

where $L_{e_{2}}$ is the Kalman filter gain matrix

Once an estimate of the disturbance has been provided by either Kalman filter, the disturbance vector $D$ can easily be formed by iterating (10) or (11) over the prediction horizon.

The overall controller structure is summarized graphically in Figure 1.

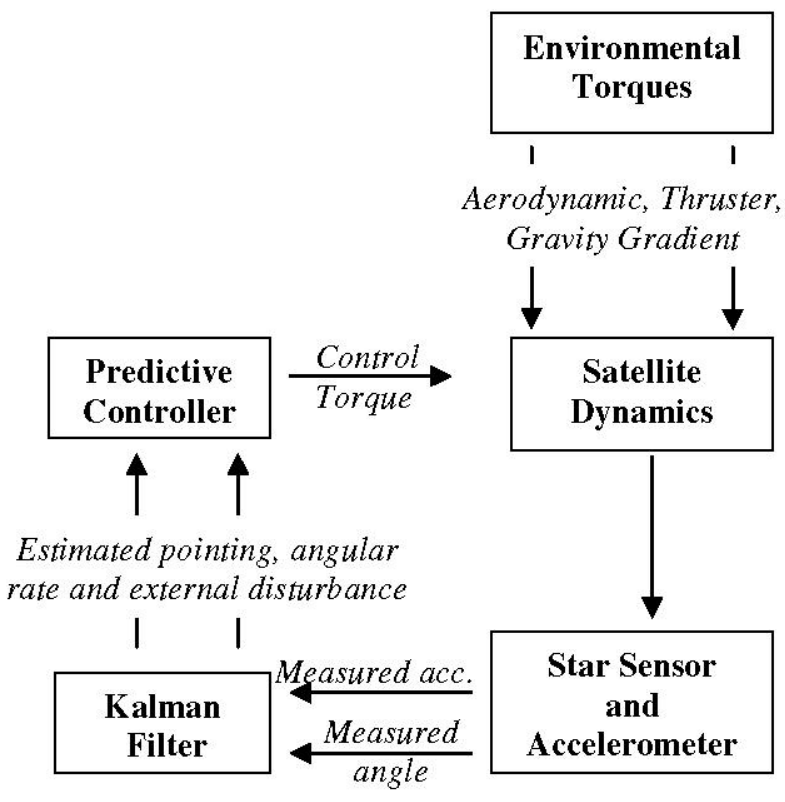

Figure 1 - Controller Structure

\section{B. Controller Tuning}

A predictive controller is designed to minimize the performance index in (6) subject to the constraint in (7). A sampling interval of $10 \mathrm{~s}$ is chosen, with prediction and control horizons of 20 time steps. The state and control weighting matrices are defined as

$$
\begin{aligned}
& Q=\operatorname{diag}\left(\left[\begin{array}{llllll}
50 & 2 e 4 & 1 & 1 e 9 & 1 e 7 & 1 e 9
\end{array}\right]\right) \\
& R=\operatorname{diag}\left(\left[\begin{array}{llll}
6 e 7 & 4 e 6 & 1 e 7
\end{array}\right] .\right.
\end{aligned}
$$

In order to achieve suitable estimation accuracy, the Kalman filter is implemented at a higher sampling frequency of $10 \mathrm{~Hz}$. 


\section{SIMULATION}

\section{A. GOCE Satellite}

Set for launch in 2008, the Gravity Field and Steady State Ocean Circulation Explorer (GOCE) satellite is part of the ESA living planet programme. Due to its low orbit altitude of just $270 \mathrm{~km}$ the GOCE satellite is subject to significant external disturbances and provides an excellent benchmark for the proposed control strategies. GOCE operates at an orbital inclination of $96^{\circ}$ and has inertia matrix $J=\operatorname{diag}\left(\left[\begin{array}{lll}152 & 2690 & 2652\end{array}\right]\right)$. For further information regarding GOCE the interested reader may consult [3].

\section{B. Controller Performance}

The satellite is initialized at pointing angles of $1^{\circ}$ about each axis. The satellite is subject to aerodynamic disturbances from the upper atmosphere and disturbance torques from the onboard thruster assembly. All simulations are carried out on a high-fidelity non-linear model provided by ESA.
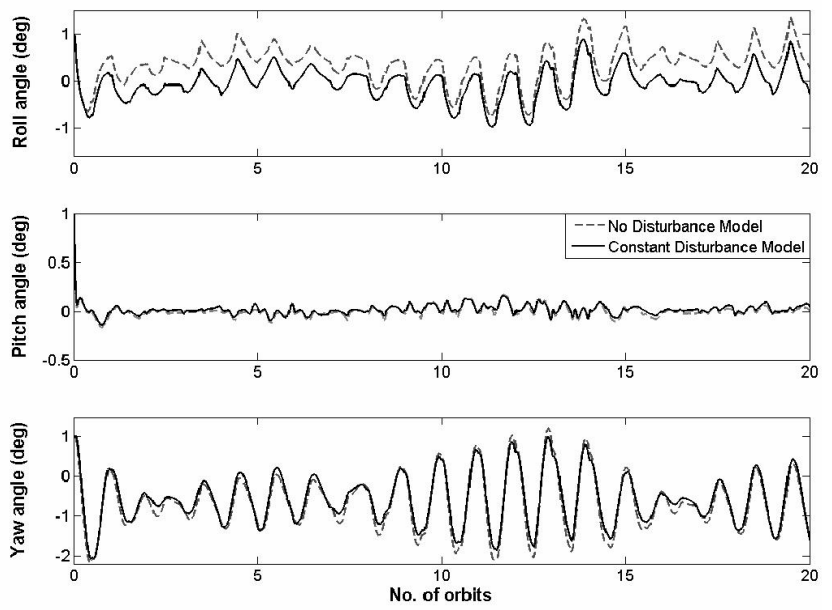

Figure 2 - Controller response with and without disturbance modeling
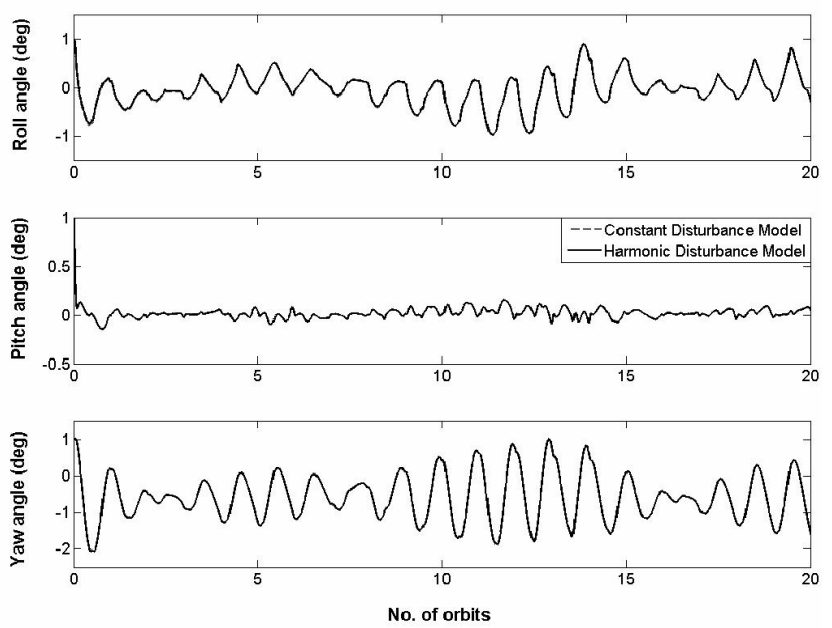

Figure 3 - Controller response with constant and harmonic disturbance modeling
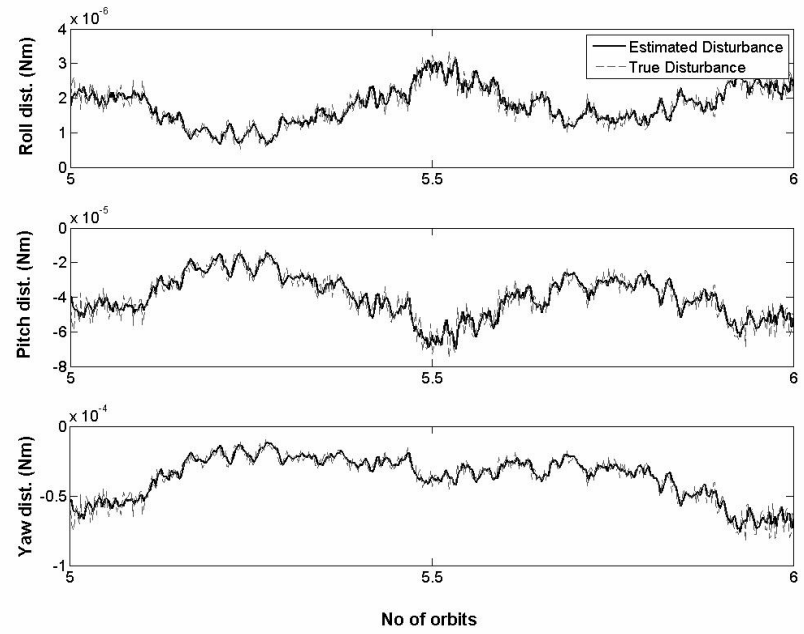

Figure 4 - Performance of Kalman filter with $\omega_{0}$ disturbance model

\section{DISCUSSION}

The simulation results clearly show the benefits of including a disturbance model within the predictive controller. Figure 2 shows that when the disturbance is estimated and assumed constant over the prediction horizon, performance is noticeably improved about the roll and yaw axes when compared to the standard MPC approach. This is achieved with minimal increase to the complexity of the controller. A lesser improvement is seen in the pitch axis, however due to the nature of the Earth's magnetic field this axis is fully controllable and good performance is achieved regardless of disturbance model.

Attempts to increase the complexity of the disturbance model seem to have little effect on the performance of the controller. Figure 3 shows the time response using the constant and harmonic disturbance models to be almost indistinguishable. There are two probable reasons for this. Firstly, the prediction horizon covers a total of 200 s which is a relatively short time when compared to the orbital period of 5400s. Although the disturbance does change over the prediction horizon, the change is relatively small. Secondly, although the Kalman filter provides a good mean estimate of the disturbance, high frequency fluctuations due to thruster noise or air density variation cannot be accurately estimated. Over a short period it is these terms that dominate the disturbance changes and cannot be predicted regardless of the disturbance model complexity.

Figure 4 shows that the Kalman filter provides very good estimates of the mean external disturbances. These estimates provide very useful data within the control law and allow for the improvement in performance seen in Figure 2. It is however clear that the $\omega_{0}$ harmonic model of the disturbances does not fully describe the true external environment and that higher frequencies exist within the system. The possibility exists to extend the complexity of the 
disturbance model further, assuming a higher order Fourier representation of the external disturbances. This possibility is to be investigated in the future, but due to the reasons already discussed it is unlikely to offer any significant benefits while largely increasing the complexity of the observer.

\section{CONCLUSIONS}

The use of a finite horizon controller with disturbance model has been investigated to improve the attitude control of a magnetically actuated satellite. Inclusion of a very simple disturbance model and state estimator demonstrates noticeable performance improvement about all 3 satellite axes when compared to standard predictive control. Studies have been carried out to investigate the benefits of increasing the complexity of the disturbance model to assume harmonic variation at the orbital frequency. This is shown to offer no benefit to overall controller performance, but does increase the complexity of the observer design. The possibility exists to further increase the complexity of the disturbance model to include higher order harmonics. Although this is to be investigated it is unlikely to offer any significant benefits due to the slow nature of the attitude dynamics and unpredictability of several aspects of the external environment.

\section{ACKNOWLEDGMENT}

The authors would like to thanks Christian Philippe and Denis Fertin for their useful comments and provision of the GOCE simulator.

\section{REFERENCES}

[1] Martel, F., Pal, P., Psiaki, M. "Active Magnetic Control System for Gravity Gradient Stabilized Spacecraft," In $2^{\text {nd }}$ Annular AIAA/USU Conference on Small Satellites, Logan, Utah, USA, 1988.

[2] Arduini, C., Baiocco, P. "Active Magnetic Damping Attitude Control for Gravity Gradient Stabilized Spacecraft," Journal of Guidance and Control, 20(1):117-122, 1997.

[3] Sechi, G., André, G., Andreis, D., Saponara, M. "Magnetic Attitude Control of the GOCE Satellite," In Proceedings of the $6^{\text {th }}$ International ESA Conference on Guidance, Navigation and Control Systems, Loutraki, Greece, 2006.

[4] Psiaki, M. "Magnetic Torquer Attitude Control via Asymptotic Periodic Linear Quadratic Regulation," In AIAA Guidance, Navigation, and Control Conference, Denver, Colorado, USA, 2000.

[5] Pittelkau, M. "Optimal periodic control for spacecraft pointing and attitude determination," Joumal of Guidance, Control and Dynamics, Vol. 16, No. 6, pp. 1078-1084, 1993.

[6] Lovera, M., De Marchi, E., Bittani, S. "Periodic Attitude Control Techniques for Small Satellite With Magnetic Actuators," IEEE Transactions on Control Systems Technology, 10(1):90-95, 2002.

[7] Silani, E., Lovera, M. "Magnetic Spacecraft Attitude Control: A Survey and Some New Results," Control Engineering Practice 13:357-371, 2005.

[8] Wood, M., Chen, W-H., Fertin, D. "Model Predictive Control of Low Earth Orbiting Spacecraft with Magneto-torquers," Proceedings of IEEE International Conference on Control Applications, Munich, 2006

[9] Krogstad, T., Gravdahl, J. Tondel, P. "Explicit Model Predictive Control of a Satellite With Magnetic Torquers," In Proceedings of the
2005 IEEE International Symposium on, Mediterranean Conference on, Control and Automation, 2005. 